BROWN-HET-1205

hep-th/9910

\title{
Non-BPS Branes on a Calabi-Yau Threefold and
}

\section{Bose-Fermi Degeneracy}

\author{
Mihail Minailescu ${ }^{b 1}$, Kyungho OH ${ }^{a}$ and Radu TataR ${ }^{b 2}$ \\ ${ }^{a}$ Dept. of Mathematics, University of California, Santa Barbara, CA 93106, USA \\ and \\ Dept. of Mathematics, University of Missouri-St. Louis, St. Louis, MO 63121, USA \\ oh@math.umsl.edu \\ ${ }^{b}$ Dept. of Physics, Brown University, Providence, RI 02912, USA \\ 1 mm@het.brown.edu \\ 2 tatar@het.brown.edu
}

\begin{abstract}
We consider the spectrum of open strings for non-BPS D-brane configuration in type II string theory on a Calabi-Yau threefold. In general, there is no degeneracy between bosonic and fermionic states. However we find special values for the moduli space of Calabi-Yau threefolds there are non-BPS brane configurations which have an exact degeneracy between bosonic and fermionic states. For these values there is no force between pairs of non-BPS D-branes. This gives rise to a possibility of building diverse nonsupersymmetric gauge field theories on the brane world-volume. We use the approach recently elaborated by Gaberdiel and Sen.
\end{abstract}




\section{Introduction}

Our current understanding of string theory suggests that there are five consistent superstring models: type IIA, type IIB, type I , SO(32) and $E_{8} \times E_{8}$ heterotic and a unique eleven dimensional supergravity theory, all of them being perturbative expansions of an underlying theory, $\mathrm{M}$ theory. We know how to connect various corners of $\mathrm{M}$ theory moduli space corresponding to different string theories but we do not have a clear picture of what happens in the intermediate region.

The main tool to connect various theories are D-branes which are solitons with the property that the open strings can end on them. They are supersymmetric solutions of the equations of motion and sit in the short representations of the supersymmetry algebra (BPS states). The last property allows the D-branes to remain stable when one goes from a weak to a strong coupling constant.

An important question is what happens when we try to analyze the non-perturbative duality beyond the BPS level. Are the non-BPS states stable? If yes, how do we map them in duality?

The existence of stable non-BPS D-branes has significant ramifications on the gauge field theories on the D-brane world-volume. As in the case of supersymmetric field theories studied from supersymmetric brane configurations (for a detailed review see [1]), nonsupersymmetric gauge field theory can be studied from the non-BPS D-branes if there is no interacting force between them.

The study of non-BPS states has started with the work of Sen [2, 3, 4, 5, 6, 7] who built non-BPS D-branes by considering pairs of branes-antibranes and applying an orbifold GSO projection. In [5, 6] the heterotic/Type I duality was tested at non-BPS level by identifying non-BPS and stable states which are mapped into each other. In [10, 11, 12, 13, 14, 15], the D-branes were considered as boundary states in the closed string theory that satisfy different projections and conservation conditions. For detailed reviews of non-BPS D-branes and their use see [16, 17, 18. When the BPS D-branes are regarded as tachyonic kinks solutions on non-BPS D-branes of higher dimensions, descent relations between BPS and non-BPS states are obtained and this allows to identify the D-brane charges with elements of K-theory as in [20, 21, 22, 23, 24, 25, 26, 27.

In [19], non-supersymmetric systems of D-branes in type IIA/IIB string theory compactified on an orbifold K3 were considered. For special points in the moduli space of the K3 orbifold an exact Bose-Fermi degeneracy in the open string spectrum was obtained. This means that up to one loop in open string theory, the D-branes do not exert any force between them.

In this paper we go one step further by considering non-supersymmetric systems of D-branes compactified on a Calabi-Yau threefold in type IIA/IIB string theory. This will give rise to a four dimensional non-supersymmetric theory. The Calabi-Yau threefold considered in this paper is a quotient of a product of the K3 orbifold and a two dimensional torus. This particular Calabi-Yau orbifold has been considered in [28, 7]. The D-branes 
we consider wrap cycles of this Calabi-Yau threefold and are also extended in some of the non-compact directions. In T-dual description, these D-branes wrap non-supersymmetric cycles on a Calabi-Yau threefold. By using these brane configuration, we are going to study the one-loop partition function with open strings and the tree-level partition function with closed strings. We show that both these partition functions vanish at special points of the moduli space of Calabi-Yau threefold. This implies that the branes do not exert any force between them.

The content of this paper is as follows: in section 2 we describe a Calabi-Yau threefold that we are interested in and its corresponding cycles. In Section 3 we describe the nonBPS states and we calculate the one-loop partition function by using open strings ending on the corresponding non-BPS D-branes. In section 3 we calculate the tree level partition function from a closed string approach. We find the critical radii of the compact directions for which the partition function vanishes.

\section{Cycles on a Calabi-Yau threefold}

We consider D branes wrapped on non-supersymmetric 2-cycle or 3-cycle of a Calabi-Yau threefold. The Calabi-Yau threefold we are concerned is a quotient of $T^{6}$ by $\mathbf{Z}_{2} \times \mathbf{Z}_{2}$. This was considered in [28, 7]. Let $x^{4}, \ldots, x^{9}$ be the coordinates of $T^{6}$ with radii $R_{4}, \ldots, R_{9}$. The action of $\mathbf{Z}_{2} \times \mathbf{Z}_{2}$ will be generated by the actions $\mathcal{I}_{4}, \mathcal{I}_{4}{ }^{\prime}$ such that

$$
\begin{aligned}
& \mathcal{I}_{4}:\left(x^{4}, \ldots x^{9}\right) \rightarrow\left(x^{4}, x^{5},-x^{6},-x^{7},-x^{8},-x^{9}\right) \\
& \mathcal{I}_{4}{ }^{\prime}:\left(x^{4}, \ldots x^{9}\right) \rightarrow\left(-x^{4},-x^{5},-x^{6}+\pi R_{6},-x^{7}, x^{8}+\pi R_{8}, x^{9}\right) .
\end{aligned}
$$

Thus we compactify type IIA string theory $T^{6}$ and mod out the theory by the $\mathbf{Z}_{2} \times \mathbf{Z}_{2}$ symmetry. By modding out by the first action $\mathcal{I}_{4}$ on $T^{6}$, we obtain $T^{2} \times T^{4} / \mathbf{Z}_{2}$. There are 16 fixed points on $T^{4}$ under $\mathbf{Z}_{2}$ induced by $\mathcal{I}_{4}$. Thus there are 16 tori which form the singular locus of $T^{2} \times T^{4} / \mathbf{Z}_{2}$. By blowing up the 16 fixed points on $T^{4} / \mathbf{Z}_{2}$, we obtain a product of a torus $T^{2}$ and a $\mathrm{K} 3$ surface. Now we return to the singular space $T^{2} \times T^{4} / \mathbf{Z}_{2}$ and note that $\mathcal{I}_{4}{ }^{\prime}$ induces an involution on $T^{2} \times T^{4} / \mathbf{Z}_{2}$ without fixed points. This is because $x^{8}$-coordinates are shifted by $\pi R_{8}$. By taking a further quotient of $T^{2} \times T^{4} / \mathbf{Z}_{2}$ by $\mathcal{I}_{4}{ }^{\prime}$, we obtain a Calabi-Yau orbifold. The singular locus on this Calabi-Yau orbifold is the images of 16 fixed tori under $\mathcal{I}_{4}{ }^{\prime}$. Thus the singular locus consists of 8 tori.

To construct a non-BPS D-brane configuration on this Calabi-Yau orbifold, we have to begin with a non-BPS D-brane configuration wrapping on cycles on $T^{6}$ which are invariant under $\mathcal{I}_{4}$ and $\mathcal{I}_{4}{ }^{\prime}$. The $\mathcal{I}_{4}$ and $\mathcal{I}_{4}{ }^{\prime}$ invariant cycles on $T^{6}$ are the images of $\mathcal{I}_{4}{ }^{\prime}$ invariant cycles on $T^{2} \times T^{4} / \mathbf{Z}_{2}$ under the quotient map $\mathcal{I}_{4}{ }^{\prime}$. We may obtain $\mathcal{I}_{4}{ }^{\prime}$ invariant two or three cycles on $T^{2} \times T^{4} / \mathbf{Z}_{2}$ in the following manner. Let $C$ be a 1-cycle on $T^{2}$ and $S$ be a 2-cycle on $T^{4} / \mathbf{Z}_{2}$. We denote the images of $C$ under $\mathcal{I}_{4}{ }^{\prime}$ by $C^{\prime}$ and the images of $S$ under $\mathcal{I}_{4}{ }^{\prime}$ by $S^{\prime}$ respectively. Then $S+S^{\prime}$ and $C \times S+C^{\prime} \times S^{\prime}$ are $\mathcal{I}_{4}{ }^{\prime}$ invariant two and three 
cycles on $T^{2} \times T^{4} / \mathbf{Z}_{2}$ respectively. Then by taking a quotient by $\mathcal{I}_{4}{ }^{\prime}$ we obtain cycles on the Calabi-Yau orbifold $T^{2} \times T^{4} / \mathbf{Z}_{2} \times \mathbf{Z}_{2}$.

\section{Open String Approach}

We want to study the case of a non-BPS state of type IIA string theory on the CalabiYau orbifold constructed in $\S 2$. In order to have a non-BPS D-brane in type IIA on the Calabi-Yau orbifold, we need to have an odd number of tangential directions of the D-brane along $T^{6}$. T Then we take a union of a non-BPS D-brane and its transformation under $\mathcal{I}_{4}^{\prime}$ action.

More specifically, we start with a D1 string wrapped along the compact $x^{9}$-th direction located at the fixed points of the action $\mathcal{I}_{4}$ which, after T-duality, can be identified with a D-brane wrapped on a non-supersymmetric 2-cycle. In general, the p-branes we deal with in this paper are extended along the non-compact directions except the $x^{9}$-th direction. The non-compact directions are transverse to the Calabi-Yau orbifold. Thus we have a $p$-brane $D$ at

$$
x^{4}=x^{5}=x^{6}=x^{7}=x^{8}=0,
$$

and its transformation $D^{\prime}$ at

$$
x^{4}=x^{5}=0, x^{6}=\pi R_{6}, \quad x^{7}=0, \quad x^{8}=\pi R_{8} .
$$

The pair $D, D^{\prime}$ gives a non-BPS D-brane configuration on the $T^{6}$ which is invariant under $\mathcal{I}_{4}$ and $\mathcal{I}_{4}{ }^{\prime}$. We will also discuss the case $x_{4} \neq 0, x_{5} \neq 0$ in the second part of this section.

Now We will calculate the open string partition function and show it vanishes at the critical radii in $\left(x^{6}, x^{7}, x^{8}, x^{9}\right)$ direction:

$$
Z=\int \frac{d t}{2 t} \operatorname{Tr}_{N S-R}\left(e^{-2 t H_{o}} \mathcal{P}\right)
$$

where NS and R denote Neveu-Schwarz and Ramond sectors respectively. $\mathcal{P}$ is a projection operator which ensures that the Fock space is physical and $H_{o}$ is the open string Hamiltonian:

$$
H_{o}=\pi \vec{p}^{2}+\frac{1}{4 \pi} \vec{w}^{2}+\pi \sum_{\mu=0,3, \ldots 9}\left[\sum_{n=1}^{\infty} \alpha_{-n}^{\mu} \alpha_{n}^{\mu}+\sum_{r>0} r \psi_{-r}^{\mu} \psi_{r}^{\mu}\right]+\pi C_{o},
$$

where $\vec{p}$ denotes the open string momentum along the directions for which the string has Neumann $(\mathrm{N})$ boundary conditions at both ends, and $\vec{w}$ denotes the winding modes along the directions for which both ends obey Dirichlet (D) boundary conditions. $\alpha_{n}^{\mu}$ and $\psi_{r}^{\mu}$ are the bosonic and fermionic oscillators satisfying the usual commutation and

\footnotetext{
${ }^{*}$ We would like to thank professor Ashoke Sen for explaining to us this and other details of this section.
} 
anti-commutation relations. The index $n$ always will be integer values, whereas the index $r$ will be integer (integer $+\frac{1}{2}$ ) values in the R (NS) sector for directions satisfying the same boundary condition at both ends of the open string (i.e. both Neumann $(\mathrm{N})$ or both Dirichlet (D)). For directions satisfying different boundary conditions at the two ends of the open string (one $\mathrm{D}$ and one $\mathrm{N}$ ) the index $n$ will be integer $+\frac{1}{2}$ values and the index $r$ will be integer $+\frac{1}{2}$ (integer) values in the $\mathrm{R}(\mathrm{NS})$ sector. The normal ordering constant $C_{o}$ vanishes in the R-sector and is equal to $-\frac{1}{2}+\frac{s}{8}$ in the NS sector (in $\alpha^{\prime}=1$ units) where $s$ denotes the number of coordinates satisfying $\mathrm{D}-\mathrm{N}$ boundary conditions.

For each D brane we have two different Chan-Paton factors - the $2 \times 2$ identity matrix I and the Pauli matrix $\sigma_{1}$. Because we have 2 D-branes, there are four Chan Paton sectors and these four sectors are $D D, D^{\prime} D^{\prime}, D D^{\prime}, D^{\prime} D$ respectively. In order to calculate the open string partition function, we need to calculate the contribution from sectors which are invariant under $\mathcal{I}_{4}{ }^{\prime}$ But the action of $\mathcal{I}_{4}{ }^{\prime}$ just exchanges the $D D$ sector with $D^{\prime} D^{\prime}$ sector and the $D D^{\prime}$ sector with the $D^{\prime} D$ sector so that its action just reduces the number of open strings by half. Thus it is enough to consider the contribution from the $D D^{\prime}$ and $D D$ sectors. As discussed in [19] the combined contribution of the Chan Paton factors from NS sector states is:

$$
\int \frac{d t}{2 t} \operatorname{tr}_{N S}\left(e^{-2 t H_{o}} \frac{1+(-1)^{F} \cdot g}{2}\right)
$$

and the contribution from the $\mathrm{R}$ sector is

$$
\int \frac{d t}{2 t} \frac{1}{2} \operatorname{tr}_{R}\left(e^{-2 t H_{o}}\right)
$$

where all the traces are over the Fock space of oscillators. We introduce some functions to simplify the presentation,

$$
\begin{aligned}
q & =e^{-\pi t} \\
\theta(R) & =\sum_{n \in \mathbf{Z}} q^{2 R^{2} n^{2}}
\end{aligned}
$$

We also introduce Dedekind $\eta$-type functions ,

$$
\begin{aligned}
& f_{1}(q)=q^{\frac{1}{12}} \prod_{n=1}^{\infty}\left(1-q^{2 n}\right), \\
& f_{2}(q)=\sqrt{2} q^{\frac{1}{12}} \prod_{n=1}^{\infty}\left(1+q^{2 n}\right) \\
& f_{3}(q)=q^{-\frac{1}{24}} \prod_{n=1}^{\infty}\left(1+q^{2 n-1}\right), \\
& f_{4}(q)=q^{-\frac{1}{24}} \prod_{n=1}^{\infty}\left(1-q^{2 n-1}\right) .
\end{aligned}
$$


Before calculating the contributions to the partition function we need to make some observations. The $D$ brane is located at $x^{6}=0, x^{8}=0$, the $D^{\prime}$ brane is located at $x^{6}=\pi R_{6}, x^{8}=\pi R_{8}$, and the distance between them is $\pi \sqrt{\left(R^{6}\right)^{2}+\left(R^{8}\right)^{2}}$. To take care of the winding modes computation of the open string properly, one needs to choose new rotated coordinates $x^{6^{\prime}}, x^{8^{\prime}}$ for the $\left(x^{6}, x^{8}\right)$ plane. The new coordinates will be given by

$$
x^{6^{\prime}}=R_{8} x^{6}-R_{6} x^{8}, \quad x^{8^{\prime}}=R_{6} x^{6}+R_{8} x^{8} .
$$

In new coordinates, two dimensional torus in the $\left(x^{6}, x^{8}\right)$-direction will be of radii $\sqrt{R_{6}^{2}+R_{8}^{2}}$ in $x^{6^{\prime}}$-direction and $\frac{R_{6} R_{8}}{\sqrt{R_{6}^{2}+R_{8}^{2}}}$ in $x^{8^{\prime}}$-direction. In view of this, we adopt a new notation

$$
R_{6}^{\prime}=\frac{\sqrt{R_{6}^{2}+R_{8}^{2}}}{2}, \quad R_{8}^{\prime}=\frac{R_{6} R_{8}}{\sqrt{R_{6}^{2}+R_{8}^{2}}} .
$$

The denominator 2 in the definition of $R_{6}^{\prime}$ reflects the fact that the string is stuck between two D branes $\pi \sqrt{\left(R^{6}\right)^{2}+\left(R^{8}\right)^{2}}$ apart. Note that the winding modes of the open string will be in in the $\left(x^{4}, x^{5}, x^{6^{\prime}}, x^{7}, x^{8^{\prime}}\right)$ directions and the momenta of the string will be in the $x^{9}$ direction because $\mathrm{D}$ branes impose Neumann boundary conditions along the $x^{9}$ direction.

We now evaluate the terms from different sectors. We take first the untwisted NS sector. All the traces are taken over the full Fock space of the open string and includes a sum (integral) over various various momentum and winding numbers and a sum over Chan Paton sectors. If we consider the $D D$ sector, then the summation over the winding modes in the $x^{6^{\prime}}$ directions will involve only integer winding modes i.e. even-integers in terms of $R_{6}^{\prime}$. This is because strings start and end on the same $\mathrm{D}$ brane and thus cover $R_{6}^{\prime}$ an even number of times. If we consider the $D D^{\prime}$ sector, then we sum over odd-integers in terms of $R_{6}^{\prime}$ because the strings starting and ending on different D-branes cover an odd number of times $R_{6}^{\prime}$. If we consider both sectors then we obtain a summation over all integer winding numbers, thus the term which corresponds to winding modes in the $x^{6^{\prime}}$ direction is $\theta\left(R_{6}^{\prime}\right)$. The same discussion holds for the untwisted sector. If we now consider the twisted $\mathrm{R}$ sector, it can come only from strings ending on the same brane, so it comes only from the $D D$ sector. Considering all of the above we obtain:

$$
\begin{aligned}
\operatorname{tr}_{N S}\left(e^{-2 t H_{o}}\right) & =A(2 t)^{-\frac{p}{2}}\left(\frac{f_{3}(q)}{f_{1}(q)}\right)^{8} \theta\left(R_{9}^{-1}\right) \prod_{i=4,5,7} \theta\left(R_{i}\right) \prod_{i=6,8} \theta\left(R_{i}^{\prime}\right) \\
t r_{N S}\left(e^{-2 t H_{o}}(-1)^{F} \cdot g\right) & =-4 A(2 t)^{-\frac{p}{2}}\left(\frac{f_{3}(q) f_{4}(q)}{f_{1}(q) f_{2}(q)}\right)^{4} \prod_{i=4,5} \theta\left(R_{i}\right) \\
\operatorname{tr}_{R}\left(e^{-2 t H_{o}}\right) & =A(2 t)^{-\frac{p}{2}}\left(\frac{f_{2}(q)}{f_{1}(q)}\right)^{8} \theta\left(R_{9}^{-1}\right) \prod_{i=4,5,7} \theta\left(R_{i}\right) \prod_{i=6,8} \theta\left(R_{i}^{\prime}\right) .
\end{aligned}
$$


where $A$ is the normalized $p$-dimensional volume of the brane in the non-compact directions. In (3.15) and (3.17) we use the fact that we have six compact coordinates and we considered the winding modes on five of them and the momentum in the $x^{9}$ compact direction. In formula (3.16) we do not have any contribution from the winding modes on the $x^{6^{\prime}}, x_{7}, x_{8}{ }^{\prime}, x^{9}$ directions but we have contribution from the winding modes on the $x^{4}, x^{5}$ directions because they are not acted upon by $\mathcal{I}_{4}$ so they survive the projection.

We now add the contributions from equations (3.5) and (3.6) to obtain the following partition function:

$Z=\int \frac{d t}{4 t} A(2 t)^{-\frac{p}{2}}\left[\left(\frac{f_{4}(q)}{f_{1}(q)}\right)^{8} \theta\left(R_{9}^{-1}\right) \prod_{i=4,5,7} \theta\left(R_{i}\right) \prod_{i=6,8} \theta\left(R_{i}^{\prime}\right)-4\left(\frac{f_{3}(q) f_{4}(q)}{f_{1}(q) f_{2}(q)}\right)^{4} \prod_{i=4,5} \theta\left(R_{i}\right)\right]$.

Here we used the identity

$$
f_{3}(q)^{8}-f_{2}(q)^{8}=f_{4}(q)^{8}
$$

We want to know when this amplitude becomes zero. First we observe that the term involving $x^{4}$ and $x^{5}$ factors out and therefore there is no conditions for the radius of the torus in the $x^{4}$ and $x^{5}$ directions. For the other 4 directions, if $R_{7}=R_{6}^{\prime}=R_{8}=1 / \sqrt{2}$ and $R_{9}=\sqrt{2}$ i.e. at the critical radii, then the contribution of the winding modes and momenta will be

$$
\theta\left(R_{7}\right) \theta\left(R_{9}^{-1}\right) \theta\left(R_{6}^{\prime}\right) \theta\left(R_{8}^{\prime}\right)=\left(\sum_{n \in \mathbf{Z}} q^{n^{2}}\right)^{4}=\left(\sqrt{2} \frac{f_{1}(q) f_{3}(q)}{f_{2}(q) f_{4}(q)}\right)^{4}
$$

where for the last equality we used the sum and the product representation of the Jacobi $\vartheta$-function $\vartheta_{3}(0 \mid \tau)$ [33]

$$
\vartheta_{3}(0 \mid \tau)=\sum_{n \in \mathbf{Z}} q^{n^{2}}=\prod_{n=1}^{\infty}\left(1-q^{2 n}\right)\left(1+q^{2 n-1}\right)^{2}=f_{1}(q) f_{3}^{2}(q),
$$

and the identity

$$
f_{4}(q) \frac{1}{\sqrt{2}} f_{2}(q) f_{3}(q)=1
$$

Taking into account our definition (3.14), the original critical radii are $R_{6}=R_{8}=1$.

By plugging now (3.20) in (3.18), we obtain that the contribution of the $D D^{\prime}$ sector to the partition function is zero at the critical radii $R_{7}=R_{6}^{\prime}=R_{8}^{\prime}=1 / \sqrt{2}, R_{9}=\sqrt{2}$. We see that the critical radius condition has to be only imposed on the $6,7,8$ and 9 directions, the 4 and 5 directions having arbitrary radii. 
We need to check whether the critical radii give a tachyon free model. We do this by calculating the mass of the NS sector ground state of the $D D^{\prime}$ string, this represents a scalar with $m^{2}=\left(R_{8}^{2}+R_{6}^{2}\right) / 4-(1 / 2)$ because the $D$ and $D^{\prime}$ are separated only in these two directions. Thus at the critical radius $\sqrt{R_{8}^{2}+R_{6}^{2}}=\sqrt{2}$ the mass is zero so there is no tachyonic scalar. Since there are no tachyonic fermions, we do not have a tachyonic component.

The critical radii are the ones where we have Bose-Fermi degeneracy at the massless level. Because besides the compact directions we have $\left(x_{1}, x_{2}, x_{3}\right)$ non-compact directions, the branes can be at different positions in those directions. But this just introduces an overall extra factor of $q^{r^{2} / 2 \pi^{2}}$ in front of the partition function which is associated with the tension of the open string stretched over a distance $r$. The potential energy depends on the non-compact direction $r$ and is equal to the negative of the partition function. It vanish identically for any value of $r$ and is a monotonically decreasing function of $r$. It is positive definite because the partition function becomes negative when we leave the $R_{7}=\frac{1}{\sqrt{2}}, R_{6}=R_{8}=1, R_{9}=\sqrt{2}$ critical radii case. Therefore for $R_{7}>\frac{1}{\sqrt{2}}, R_{6}>1, R_{8}>$ $1, R_{9}<\sqrt{2}$, the interaction between branes is repulsive at all non-compact distances.

We have discussed the case when the $D$ and $D^{\prime}$ branes are at $x^{4}=x^{5}=0$. What happens when the $\mathrm{D}$ brane is displaced from this point to $x^{4}=b_{4}, x^{5}=b_{5}$ where $b_{4}$ or $b_{5}$ is non-zero? Then the $\mathrm{D}^{\prime}$ must be displaced to $x^{4}=-b_{4}, x^{5}=-b_{5}$ in order to have an $\mathcal{I}_{4}{ }^{\prime}$ invariant configuration. In this case the mass of the NS sector ground state is

$$
m^{2}=\frac{\left(2 b_{4} / \pi\right)^{2}+\left(2 b_{5} / \pi\right)^{2}+R_{6}^{2}+R_{8}^{2}}{4}-\frac{1}{2},
$$

so the condition of a tachyon free theory is

$$
4\left(b_{4} / \pi\right)^{2}+4\left(b_{5} / \pi\right)^{2}+R_{6}^{2}+R_{8}^{2}=2 .
$$

Here we assume that $0<b_{4}<\pi R_{4}$ and $0<b_{5}<\pi R_{5}$ But, by considering the winding modes in $\left(x_{4}, x_{5}\right)$ directions from the winding modes in $\left(x_{6}, x_{7}, x_{8}\right)$ separately, one can see that the partition function remains to be the same as in our main case (3.18) because $\mathcal{I}_{4}$ projection does not act on the $\left(x_{4}, x_{5}\right)$ directions and again $\mathcal{I}_{4}{ }^{\prime}$ just exchanges the different sectors. This implies that $R_{6}^{2}+R_{8}^{2}$ must be 2 if we want the partition function to vanish. We conclude that $b_{4} b_{5}$ must be zero in order to have a tachyon free theory.

\section{Closed String Approach}

We now compare the results of the previous section with the ones obtained from a closed string theory point of view, where the D branes are viewed as boundary states [29, 30, [31, 32]. We want firstly to identify the boundary states describing the pair of D-branes wrapped on one direction of the orbifold. Before the $\mathcal{I}_{4}{ }^{\prime}$ projection, the boundary state 
that represents the non-BPS brane is of the form [11]:

$$
\begin{aligned}
|\widetilde{D} p, \mathbf{a}, \mathbf{b}, \mathbf{c}\rangle=\frac{1}{\sqrt{2}}\left(|B p, \mathbf{a}, \mathbf{b}, \mathbf{c},+\rangle_{N S N S}^{U}-|B p, \mathbf{a}, \mathbf{b}, \mathbf{c},-\rangle_{N S N S}^{U}\right) \\
+\frac{1}{2} \epsilon\left(|B p, \mathbf{a}, \mathbf{b}, \mathbf{c},+\rangle_{R R ;\left(0, \pi R_{9}\right)}^{T}+|B p, \mathbf{a}, \mathbf{b}, \mathbf{c},-\rangle_{R R ;\left(0, \pi R_{9}\right)}^{T}\right),
\end{aligned}
$$

where we use $\left(0, \pi R_{9}\right)$ to show that we have to make the summation over the RR components of the twisted sector boundary states located at $x^{9}=0$ and $x^{9}=\pi R_{9}$ respectively. $\epsilon$ is the charge of the twisted RR sector and can have values \pm . In the above formula we represents the coordinates of the noncompact directions by $\mathbf{a}$, the $x^{4}, x^{5}, x^{7}$ coordinates by b and the $x^{6}, x^{8}$ coordinates by $\mathbf{c}$ respectively. We consider only the sector with twisted $\mathrm{RR}$ charge equal to 1 . After taking the $\mathcal{I}_{4}{ }^{\prime}$ projection, we need to consider a state which is the superposition of two such non-BPS branes located at different values of $\mathbf{c}$, the first one at $\mathbf{c}_{1}=(0,0)$ and the second one at $\mathbf{c}_{2}=\left(\pi R_{6}, \pi R_{8}\right)$. This state is written as:

$$
|\widetilde{D} p\rangle=\left|\widetilde{D} p, \mathbf{a}, \mathbf{b}=0, \mathbf{c}_{1}\right\rangle+\left|\widetilde{D} p, \mathbf{a}, \mathbf{b}=0, \mathbf{c}_{2}\right\rangle
$$

where we consider both branes to be located at $\mathbf{b}=0$. This state is invariant under the $\mathcal{I}_{4}{ }^{\prime}$ because the first term is exchanged with the second one. In order to be able to map the boundary state into itself under the action of $\mathcal{I}_{4}{ }^{\prime}$ we need to chose the same $\epsilon$ parameters for both branes.

We are interested in the tree level amplitude that describes the exchange of closed strings between two such non-BPS D-branes invariant under $\mathcal{I}_{4}$ and $\mathcal{I}_{4}{ }^{\prime}$. The amplitude is given by

$$
\int_{0}^{\infty} d l\left\langle\widetilde{D} p\left|e^{-l H_{c}}\right| \widetilde{D} p\right\rangle
$$

where $H_{c}$ is the closed string Hamiltonian in light cone gauge,

$$
H_{c}=\pi \vec{p}^{2}+\frac{1}{4 \pi} \vec{w}^{2}+2 \pi \sum_{\mu=0,3, \ldots, 9}\left[\sum_{n=1}^{\infty}\left(\alpha_{-n}^{\mu} \alpha_{n}^{\mu}+\tilde{\alpha}_{-n}^{\mu} \tilde{\alpha}_{n}^{\mu}\right)+\sum_{r>0}\left(\psi_{-r}^{\mu} \psi_{r}^{\mu}+\tilde{\psi}_{-r}^{\mu} \tilde{\psi}_{r}^{\mu}\right)\right]+2 \pi C_{c} .
$$

The constant $C_{c}$ is -1 in the untwisted NSNS sector and 0 in the twisted RR sector. $\vec{p}$ and $\vec{w}$ denote the momentum and winding charges as usual.

In order to calculate the partition function, we need to introduce the corresponding boundary states in the equation (4.1).

We can now proceed to calculate the tree level closed string amplitude. Because of the action of $\mathcal{I}_{4}{ }^{\prime}$, the amplitude describing the emission and re-absorption of closed strings by the D-brane located at $\mathbf{c}_{1}$ is equal to the contribution of the D-brane located at $\mathbf{c}_{2}$ and we take only once this contribution. Also, the amplitude describing the emission of a closed string by the brane at $\mathbf{c}_{1}$ and absorption by the brane at $\mathbf{c}_{2}$ is equal with the amplitude describing the inverse process. In order to obtain the amplitude, we need to calculate 4 terms, the first between $\left|\widetilde{D} p, \mathbf{a}, \mathbf{b}=0, \mathbf{c}_{1}\right\rangle$ and $\left|\widetilde{D} p, \mathbf{a}, \mathbf{b}=0, \mathbf{c}_{2}\right\rangle$, the second one between 
$\left|\widetilde{D} p, \mathbf{a}, \mathbf{b}=0, \mathbf{c}_{2}\right\rangle$ and $\left|\widetilde{D} p, \mathbf{a}, \mathbf{b}=0, \mathbf{c}_{1}\right\rangle$, the third one between $\left|\widetilde{D} p, \mathbf{a}, \mathbf{b}=0, \mathbf{c}_{1}\right\rangle$ and itself and the fourth between $\left|\widetilde{D} p, \mathbf{a}, \mathbf{b}=0, \mathbf{c}_{2}\right\rangle$ and itself. We add all these terms and then divide by 2 because of the $\mathcal{I}_{4}{ }^{\prime}$ projection.

We need to identify the untwisted and twisted sectors. As any orbifold discussion, the twisted sectors appears at fixed points of the orbifold. Because the D-branes are at fixed points of $\mathcal{I}_{4}$, the twisted sectors are obtained from the amplitude of emission and absorption by the same brane and we only take the contribution once, as discussed before. The untwisted sector is obtained from both the amplitude of emission by one brane and absorption by the other brane and from the amplitude of emission and absorption by the same brane. Another difference which arises in our case is that we need to have in the twisted sector the winding modes coming from the two compact directions $x^{4}$ and $x^{5}$ that $\mathcal{I}_{4}{ }^{\prime}$ is not acting upon. Then the contribution from the twisted RR sector will be

$$
\frac{1}{2} \widetilde{\mathcal{N}}^{2}\left(\frac{f_{2}(q) f_{3}(q)}{f_{1}(q) f_{4}(q)}\right)^{4}\left(\prod_{i=4,5} \sum_{m \in \mathbf{Z}} e^{-l \pi m^{2} / R_{i}^{2}}\right), \quad q=e^{-2 \pi l}
$$

which is obtained from

$$
\begin{aligned}
& \frac{1}{4}\left\langle B p, \mathbf{a}, \mathbf{b}, \mathbf{c}_{1}, \pm\left.\right|_{R R} ^{T} \quad e^{-l H_{c}} \mid B p, \mathbf{a}, \mathbf{b}, \mathbf{c}_{1}, \pm\right\rangle_{R R}^{T} \\
+ & \frac{1}{4}\left\langle B p, \mathbf{a}, \mathbf{b}, \mathbf{c}_{2}, \pm\left.\right|_{R R} ^{T} \quad e^{-l H_{c}} \mid B p, \mathbf{a}, \mathbf{b}, \mathbf{c}_{2}, \pm\right\rangle_{R R}^{T} .
\end{aligned}
$$

Here the factor $1 / 4$ comes from the fact that the charge of the twisted RR sector $\epsilon$ in the boundary state decomposition (4.1) are equal for the two branes. $\widetilde{\mathcal{N}}^{2}$ in $(4.5)$ is a normalization constant to be determined later.

In order to calculate the untwisted sector contribution to the amplitude, we need to remember the form for the states appearing in the untwisted RR sector. They are:

$$
|B p, \mathbf{a}, \mathbf{b}, \mathbf{c}, \eta\rangle_{N S N S}^{U}=\mathcal{N} \int\left(\prod_{\mu \text { transverse }} d k^{\mu} e^{i \mathbf{k} \cdot \mathbf{a}}\right)\left(\prod_{i=6,8} \sum_{m \in \mathbf{Z}} e^{i m c_{i} / R_{i}}\right)|B p, \widehat{\mathbf{k}, \mathbf{m}}, \eta\rangle_{N S N S}^{U}
$$

where $\mathbf{k}$ denotes the momentum in the non-compact directions, and $m / R_{i}$ the momentum along the $i$ th compact direction and $\mathcal{N}$ is a normalization factor to be determined later. Here we are taking integration over the directions transverse to the D-brane and $|B p, \widehat{\mathbf{k}, \mathbf{m}}, \eta\rangle_{N S N S}^{U}$ denotes the coherent momentum eigenstate

$$
\begin{aligned}
|B p, \widehat{\mathbf{k}, \mathbf{m}}, \eta\rangle=\exp \left(\sum_{n=1}^{\infty}\right. & {\left[-\frac{1}{n} \sum_{\mu \in \mathcal{C}} \alpha_{-n}^{\mu} \tilde{\alpha}_{-n}^{\mu}+\frac{1}{n} \sum_{\mu \in \hat{\mathcal{D}}} \alpha_{-n}^{\mu} \tilde{\alpha}_{-n}^{\mu}\right] } \\
& \left.+i \eta \sum_{r>0}\left[-\sum_{\mu \in \mathcal{C}} \psi_{-r}^{\mu} \tilde{\psi}_{-r}^{\mu}+\sum_{\mu \in \hat{\mathcal{D}}} \psi_{-r}^{\mu} \tilde{\psi}_{-r}^{\mu}\right]\right)|B p, \widehat{\mathbf{k}, \mathbf{m}}, \eta\rangle^{(0)},
\end{aligned}
$$


where $|B p, \widehat{\mathbf{k}, \mathbf{m}}, \eta\rangle^{(0)}$ denotes the Fock vacuum labeled by the quantum numbers $\mathbf{k}, \mathbf{m}$. When we plug the states into equation (4.3), we will have a summation of the following term:

$$
1+e^{i \pi m}+e^{-i \pi m}+1=4 \cos ^{2}(\pi m / 2) .
$$

The terms in the left hand sides come from the closed strings emitted and absorbed by the same D-brane (first and fourth) and from the closed strings emitted and absorbed by different branes situated at 0 and $2 R_{6}^{\prime}$ where $R_{6}^{\prime}$ is defined in (3.14). This is non-zero only for even-integer values for $m$, therefore the summation over the winding modes on the $x_{6}^{\prime}$ direction will be over even-integer numbers. Then the contribution of the untwisted sector to the amplitude is (after taking the $\mathcal{I}_{4}{ }^{\prime}$ projection) is:

$$
\prod_{i=4,5,7} \sum_{m \in \mathbf{Z}} e^{-l \pi m^{2} / R_{i}^{2}}\left(\sum_{m \in \mathbf{Z}} e^{-l \pi m^{2} R_{9}^{2}}\right)\left(\sum_{m \in 2 \mathbf{Z}} e^{-l \pi m^{2} / 4 R_{6}^{\prime 2}}\right)\left(\sum_{m \in \mathbf{Z}} e^{-l \pi m^{2} / R_{8}^{\prime 2}}\right) \frac{f_{3}^{8}(q)-f_{4}^{8}(q)}{f_{1}^{8}(q)}
$$

where $q=e^{-2 \pi l}$.

By adding up all the contributions to the amplitude, we obtain

$$
\begin{aligned}
& \frac{1}{2} \int_{0}^{\infty} d l l^{-\frac{4-p}{2}}\left[2 \mathcal{N}^{2} \prod_{i=4,5,7} \sum_{m \in \mathbf{Z}} e^{-l \pi m^{2} / R_{i}^{2}} \sum_{m \in \mathbf{Z}} e^{-l \pi m^{2} R_{9}^{2}} \sum_{m \in 2 \mathbf{Z}} e^{-l \pi m^{2} / 4 R_{6}^{\prime 2}} \times\right. \\
\times & \left.\sum_{m \in \mathbf{Z}} e^{-l \pi m^{2} / R_{8}^{\prime 2}} \frac{f_{3}^{8}(q)-f_{4}^{8}(q)}{f_{1}^{8}(q)}-\widetilde{\mathcal{N}}^{2}\left(\frac{f_{2}(q) f_{3}(q)}{f_{1}(q) f_{4}(q)}\right)^{4} \prod_{i=4,5} \sum_{m \in \mathbf{Z}} e^{-l \pi m^{2} / R_{i}^{2}}\right],
\end{aligned}
$$

where $q=e^{-2 \pi l}$ and $\mathcal{N}, \widetilde{\mathcal{N}}$ are the normalization constants introduced for the boundary states. The power of $l$ comes from the fact that the D-brane is extended in $p$ of the 4 non-compact directions.

To determine the normalization constants, we apply a modular transformation $t=1 / 2 l$ which changes the closed string tree amplitude into an open string 1-loop amplitude. Using the properties of the $f_{i}$ functions,

$$
\begin{array}{lll}
f_{1}\left(e^{-\pi / t}\right)=\sqrt{t} f_{1}\left(e^{-\pi t}\right), & f_{2}\left(e^{-\pi / t}\right)=f_{4}\left(e^{-\pi t}\right), \\
f_{3}\left(e^{-\pi / t}\right)=f_{3}\left(e^{-\pi t}\right), & & f_{4}\left(e^{-\pi / t}\right)=f_{2}\left(e^{-\pi t}\right),
\end{array}
$$

together with the identity

$$
\sum_{m \in \mathbf{Z}} e^{-\pi l(m / R)^{2}}=\frac{R}{\sqrt{l}} \sum_{m \in \mathbf{Z}} e^{-2 t \pi(m R)^{2}}=R \sqrt{2 t} \sum_{m \in \mathbf{Z}} q^{2 m^{2} R^{2}}, \quad q=e^{-\pi t},
$$

we can express (4.11) as

$$
\begin{array}{r}
\frac{1}{2} \int_{0}^{\infty} \frac{d t}{2 t} t^{-\frac{p}{2}} 2^{\frac{6-p}{2}} R_{4} R_{5} \prod_{i=4,5} \theta\left(R_{i}\right)\left[8 \mathcal{N}^{2} \frac{R_{7} R_{6}^{\prime} R_{8}^{\prime}}{R_{9}} \theta\left(R_{7}\right) \theta\left(R_{9}^{-1}\right) \prod_{i=6,8} \theta\left(R_{i}^{\prime}\right) \frac{f_{3}^{8}(q)-f_{2}^{8}(q)}{f_{1}^{8}(q)}\right. \\
\left.-\widetilde{\mathcal{N}}^{2}\left(\frac{f_{3}(q) f_{4}(q)}{f_{1}(q) f_{2}(q)}\right)^{4}\right]
\end{array}
$$


where $q=e^{-\pi t}$. By comparing the form of the above partition function with the open string theory partition function (3.18), we set the values of normalization constants $\mathcal{N}^{2}$ and $\widetilde{\mathcal{N}}^{2}$ to be

$$
\begin{array}{r}
64 \frac{R_{4} R_{5} R_{7} R_{6}^{\prime} R_{8}^{\prime}}{R_{9}} \mathcal{N}^{2}=A, \\
8 R_{4} R_{5} \widetilde{\mathcal{N}}^{2}=4 A,
\end{array}
$$

so that the closed string amplitude becomes

$$
\begin{aligned}
\frac{A}{4} \int_{0}^{\infty} d l l^{-\frac{4-p}{2}} & \frac{1}{R_{4} R_{5}}\left(\prod_{i=4,5} \sum_{m \in \mathbf{Z}} e^{-l \pi m^{2} / R_{i}^{2}}\right)\left[\frac{R_{9}}{16 R_{7} R_{6}^{\prime} R_{8}^{\prime}}\left(\sum_{m \in \mathbf{Z}} e^{-l \pi m^{2} / R_{7}^{2}}\right)\left(\sum_{m \in \mathbf{Z}} e^{-l \pi m^{2} R_{9}^{2}}\right) \times\right. \\
& \left.\left(\sum_{m \in 2 \mathbf{Z}} e^{-l \pi m^{2} / 4 R_{6}^{\prime 2}}\right)\left(\sum_{m \in \mathbf{Z}} e^{\left.-l \pi m^{2} / R_{8}^{\prime 2}\right)}\right) \frac{f_{3}^{8}(q)-f_{4}^{8}(q)}{f_{1}^{8}(q)}-\left(\frac{f_{2}(q) f_{3}(q)}{f_{1}(q) f_{4}(q)}\right)^{4}\right]
\end{aligned}
$$

If we choose $R_{6}^{\prime}=R_{8}^{\prime}=R_{7}=1 / \sqrt{2}, R_{9}=\sqrt{2}$, then the partition function becomes zero. The corresponding values for $R_{6}, R_{8}$ are $R_{6}=R_{8}=1$. So, by using the boundary state formalism we found critical radii for which the tree level partition function vanishes.

Things might change if one takes higher-loop corrections in both open string and closed string theories.

\section{Acknowledgments}

We would like to thank M. Gaberdiel and especially A. Sen for insightful suggestions and comments. The work of K. Oh is supported in part by NSF grant PHY-9970664. K. Oh thanks Department of Mathematics at University of California, Santa Barbara for its hospitality during his stay.

\section{References}

[1] A. Giveon and D. Kutasov, Rev.Mod.Phys. 71 (1999) 983, hep-th/9802067

[2] A. Sen, JHEP 06, 007 (1998), hep-th/9803194

[3] A. Sen, JHEP 08, 010 (1998), hep-th/9805019

[4] A. Sen, JHEP 08, 012 (1998), hep-th/9805170

[5] A. Sen, JHEP 09, 023 (1998), hep-th/9808141

[6] A. Sen, JHEP 10, 021 (1998), hep-th/9809111

[7] A. Sen, JHEP 12, 021 (1998), hep-th/9812031 
[8] J. Majumder, A. Sen, JHEP 09 (1999) 004, hep-th/9906109

[9] A. Sen, hep-th/9909062

[10] O. Bergman, M. Gaberdiel, Nucl. Phys. B499 183 (1997), hep-th/9701137

[11] O. Bergman, M. Gaberdiel, Phys. Lett. B441 183 (1998), hep-th/9806155

[12] O. Bergman, M. Gaberdiel, JHEP 03, 013 (1999), hep-th/9901014

[13] O. Bergman, M. Gaberdiel, hep-th/9908126

[14] M. Gaberdiel, B. Stefanski jr., hep-th/9910109

[15] T. Dasgupta, B. Stefanski jr., hep-th/9910217

[16] A. Lerda, R. Russo, hep-th/9905006

[17] A. Sen, hep-th/9904207

[18] J. H. Schwarz, hep-th/9908014

[19] M. Gaberdiel and A. Sen, hep-th/9908060

[20] E. Witten, JHEP 12 (1998) 019, hep-th/9810188

[21] P. Horava, Adv.Theor.Math.Phys. 2 (1999) 1373, hep-th/9812135,

[22] H. Garcia-Compean, hep-th/9812226

[23] S. Gukov, hep-th/9901042

[24] K. Hori, hep-th/9902102

[25] O. Bergman, E. Gimon, P. Horava, JHEP 04 (1999) 010, hep-th/9902160

[26] K. Olsen, R. Szabo, hep-th/9907140

[27] E. Sharpe, hep-th/9902116

[28] S. Ferrara, J. Harvey, A. Strominger, C. Vafa, Phys. Lett. B361 (1995) 59, hepth/9505162

[29] J. Polchinski and Y. Cai, Nucl. Phys. B296, 91 (1988)

C. Callan, C. Lovelace, C. Nappi and S. Yost, Nucl. Phys. B308, 221 (1988)

[30] T. Onogi and N. Ishibashi, Mod. Phys. Lett. A4, 161 (1989)

N. Ishibashi, Mod. Phys. Lett. A4, 251 (1989) 
[31] C. Lovelace, Phys. Lett. B34, 500 (1971)

L. Clavelli and J. Shapiro, Nucl. Phys. B57, 490 (1973).

M. Ademollo, R. D'Auria, F. Gliozzi, E. Napolitano, S. Sciuto and P. di Vecchia, Nucl. Phys. B94, 221 (1975).

C. Callan, C. Lovelace, C. Nappi and S. Yost, Nucl. Phys. B293, 83 (1987).

M. Bianchi and A. Sagnotti, Phys. Lett. 247B, 517 (1990); Nucl. Phys. B361, 519 (1991).

P. Horava, Nucl. Phys. B327, 461 (1989)

M. Li, Nucl. Phys. B460, 351 (1996); hep-th/9510161.

H. Ooguri, Y. Oz and Z. Yin, Nucl. Phys. B477, 407 (1996); hep-th/9606112.

K. Becker, M. Becker, D. Morrison, H. Ooguri, Y. Oz and Z. Yin, Nucl. Phys. B480, 225 (1996); hep-th/9608116.

M. Kato and T. Okada, Nucl. Phys. B499, 583 (1997); hep-th/9612148.

S. Stanciu, Nucl. Phys. B526, 295 (1998); hep-th/9708166.

A. Recknagel and V. Schomerus, Nucl. Phys. B531, 185 (1998); hep-th/9712186.

J. Fuchs and C. Schweigert, Nucl. Phys. B530, 99 (1998); hep-th/9712257.

S. Stanciu and A. Tseytlin, JHEP 06, 010 (1998); hep-th/9805006.

M. Gutperle and Y. Satoh, Nucl. Phys. B543, 73 (1999); hep-th/9808080.

F. Hussain, R. Iengo, C. Nunez and C. Scrucca, Phys. Lett. B409, 101 (1997); hepth/9706186.

M. Bertolini, R. Iengo and C. Scrucca, Nucl. Phys. B522, 193 (1998); hepth/9801110.

M. Bertolini, P. Fre, R. Iengo and C. Scrucca, Phys. Lett. B431, 22 (1998); hepth/9803096.

P. Di Vecchia, M. Frau, A. Lerda, I. Pesando, R. Russo and S. Sciuto, Nucl. Phys. B507, 259 (1997); hep-th/9707068.

M. Billo, P. Di Vecchia, M. Frau, A. Lerda, I. Pesando, R. Russo and S. Sciuto, Nucl. Phys. B526, 199 (1998); hep-th/9802088.

A. Recknagel and V. Schomerus, Nucl. Phys. B545, 233 (1999); hep-th/9811237.

[32] I. Brunner, A. Rajaraman, M. Rozali, hep-th/9905024

I. Brunner, R. Entin, C. Romelsberger hep-th/9905078

D. E. Diaconescu, J. Gomis, hep-th/9906242

Arvind Rajaraman, Moshe Rozali, hep-th/9909017

D. E. Diaconescu, C. Romelberger, hep-th/hep-th/9910172

[33] A. Erdéli, W. Magnus, F. Oberhettinger and F. G. Tricomi, Higher Transcendental Functions, Vol. 2, McGraw-Hill, (1953); p. 354 ff. 